\title{
WEIGHTED INEQUALITIES OF FEFFERMAN-STEIN TYPE FOR RIESZ-SCHRÖDINGER TRANSFORMS
}

\author{
B. BongioAnni, E. Harboure AND P. QuiJano
}

Abstract. In this work we are concerned with Fefferman-Stein type inequalities. More precisely, given an operator $T$ and some $p, 1<p<\infty$, we look for operators $\mathscr{M}$ such that the inequality

$$
\int|T f|^{p} w \leqslant C \int|f|^{p} \mathscr{M} w,
$$

holds true for any weight $w$. Specifically, we are interested in the case of $T$ being any first or second order Riesz transform associated to the Schrödinger operator $L=-\Delta+V$, with $V$ a nonnegative function satisfying an appropriate reverse-Hölder condition. For the Riesz-Schrödinger transforms $\nabla L^{-1 / 2}$ and $\nabla^{2} L^{-1}$ we make use of a result due to C. Pérez where this problem is solved for classical Calderón-Zygmund operators.

Mathematics subject classification (2010): 42B20, 35J10.

Keywords and phrases: Schrödinger operator, singular integral, weights.

\section{REFERENCES}

[1] B. Bongioanni, E. Harboure And P. QuiJano, Weighted inequalities for Schrödinger type singular integrals, Journal of Fourier Analysis and Applications, 25(3):595-632, 2019.

[2] B. Bongioanni, E. Harboure And O. Salinas, Riesz transforms related to Schrödinger operators acting on BMO type spaces, J. Math. Anal. Appl., 357(1):115-131, 2009.

[3] B. Bongioanni, E. Harboure And O. Salinas, Weighted inequalities for commutators of Schrödinger-Riesz transforms, J. Math. Anal. Appl., 392(1):6-22, 2012.

[4] A. Cordoba And C. FefFerman, A weighted norm inequality for singular integrals, Studia Math., 57(1):97-101, 1976.

[5] David V. Cruz-Uribe, José Maria Martell and Carlos Pérez, Weights, extrapolation and the theory of Rubio de Francia, volume 215 of Operator Theory: Advances and Applications, Birkhäuser/Springer Basel AG, Basel, 2011.

[6] J. DZIUbAŃs Ki AND J. ZienkiewicZ, Hardy spaces $H^{1}$ associated to Schrödinger operators with potential satisfying reverse Hölder inequality, Revista Matemática Iberoamericana, 15(2):279-296, 1999.

[7] C. Fefferman and E. M. Stein, Some maximal inequalities, Amer. J. Math., 93:107-115, 1971.

[8] Tao Ma, Pablo Raúl Stinga, José L. Torrea and Chao Zhang, Regularity estimates in Hölder spaces for Schrödinger operators via a T1 theorem, Ann. Mat. Pura Appl. (4), 193(2):561589, 2014.

[9] C. PÉREZ, Weighted norm inequalities for singular integral operators, J. London Math. Soc. (2), 49(2):296-308, 1994.

[10] Z. SHEN, $L^{p}$ estimates for Schrödinger operators with certain potentials, Ann. Inst. Fourier (Grenoble), 45(2):513-546, 1995.

[11] J. Michael WiLson, Weighted norm inequalities for the continuous square function, Trans. Amer. Math. Soc., 314(2):661-692, 1989. 